\title{
Glucose transporters expression in the placental terminal villi of preeclampsia and intrauterine growth retardation complicated pregnancies
}

\author{
Elena A. Dubova, Konstantin A. Pavlov, Galina V. Kulikova, Alexander I. Shchegolev, \\ Gennady T. Sukhikh \\ Department of Pathology, Research Center for Obstetrics, Gynecology and Perinatology, Moscow, Russia; e_dubova@oparina4.ru
}

Received 23 May 2013; revised 24 June 2013; accepted 10 July 2013

Copyright (C) 2013 Elena A. Dubova et al. This is an open access article distributed under the Creative Commons Attribution License, which permits unrestricted use, distribution, and reproduction in any medium, provided the original work is properly cited.

\begin{abstract}
Introduction: Transmembrane sodium-independent glucose transporters (GLUTs) play an important role in both placental and fetal development by the providing transplacental glucose transport. Abnormal GLUT-1 and GLUT-3 placental expression could play a role in such late pregnancy complications development as preeclampsia and intrauterine growth retardation. Materials and Methods: Immunohistochemistry was performed to reveal patterns of GLUT-1 and GLUT-3 expression in the placental terminal villi compartments. Results: GLUT-1 syncytial expression in the terminal villi of severe PE cases (both with and without IUGR) was significantly lower in compare to control group. We also report about GLUT-3 both syncytial and endothelial expression in the near term and term placental terminal villi without significant difference between the study and control groups. Conclusion: other study should be performed to establish the nature of GLUT-1 downregulation in severe preeclampsia cases and whether GLUT-3 is expressed in the syncytiotrophoblast of near term and term placentas or not.
\end{abstract}

Keywords: Preeclampsia; Placenta; GLUT-1; GLUT-3

\section{INTRODUCTION}

Preeclampsia being one of the most severe pregnancy complications accounts about $12 \%$ of all maternal mortality cases [1]. Basic pathogenic events in PE development are abnormal trophoblastic invasion and spiral arteries remodeling later leading to utero-placental hypoxia
[2]. Some cases of PE are also complicated by intrauterine growth retardation [3].

Oxygen is the key substrate for normal fetal development and growth therefore hypoxia is a leading cause of fetal growth restriction. After oxygen, glucose is the most important substrate for fetal growth and the only one that can be metabolized anaerobically [4]. The second important cause of IUGR reduces glucose transfer to the fetus. Placenta is a source of alterations that lead to the reduced maternal-fetal glucose transfer. At least partially this could be due to the decreased placental glucose transporters (GLUT) expression, since previous data have suggested that transport across the basal membrane is the rate-limiting step in transplacental glucose transport [5]. GLUTs are the members of sodium-independent transmembrane proteins that provide intracellular glucose transport [6]. These molecules could play an important role in the development of fetal growth restriction in preeclampsia but its importance is not established yet. The aim of this study is to analyze glucose transporters GLUT-1 and GLUT-3 expression patterns in the terminal villi of the placentas from PE and IUGR cases.

\section{MATERIALS AND METHODS}

A cross section morphological and immunohistochemical study of 31 placentas from preeclampsia-complicated pregnancies with and without IUGR and 13 placentas from uncomplicated pregnancies was performed. Inclusion criteria were singleton pregnancy, moderate and severe PE, severe PE combined with IUGR. Other pregnancy complications, extragenital pathology and fetal abnormalities were exclusion criteria. Clinical characteristic of the study groups is presented in Table 1 .

Gross examination of all the placentas was performed. Placental weight was measured after trimming the cord and membranes. Tissue samples were obtained from the 
central parts of placental disk for microscopy and immunohistochemistry and fixed with $10 \%$ buffered formalin. H \& E stained slides were then prepared and studied microscopically.

Immunohistochemical study was performed on 3 - 4 mcm slides with GLUT-1 monoclonal mouse (1:100, Abcam, Great Britain) and polyclonal rabbit GLUT-3 (1:50, Abcam, Great Britain) antibodies.

Data are presented as mean \pm SD. Statistical analysis was performed using the Statistica 8 (StatSoft Inc, USA) software. Data were measured by analysis of variance using the Scheffe post hoc test. A value of $p<0.05$ was taken as significant.

\section{RESULTS}

\subsection{Pregnancy Characteristics}

No significant difference in maternal age and body mass index was observed between the study and control groups. Gestational age in both severe PE and severe PE with IUGR was significantly lower in compare to control and moderate PE groups ( $\mathrm{p}<0.05)$.

Newborn body weight in both severe PE and severe PE with IUGR groups was significantly lower, than in control group $(\mathrm{p}<0.05)$. No difference in placental weight was observed between moderate and severe $\mathrm{PE}$ in compare to control ( $p>0.05$ ) with only significantly lower placental weight in severe PE with IUGR group in compare to control ( $\mathrm{p}<0.05$ ). No significant difference in placental/newborn weight index was also observed ( $\mathrm{p}$ $>0.05)$.

\subsection{Placental Histology}

In 3 cases of moderate, 4 of severe PE and 5 of severe $\mathrm{PE}$ with IUGR cases sign of villous maldevelopment that

Table 1. Clinical characteristic of the study groups (Data presented as mean $\pm \mathrm{SD}$ ).

\begin{tabular}{|c|c|c|c|c|}
\hline & Control & mPE & SPE & sPE + IUGR \\
\hline $\begin{array}{c}\text { Case } \\
\text { number }\end{array}$ & 16 & 7 & 8 & 13 \\
\hline $\begin{array}{l}\text { Gestational } \\
\text { age, (weeks) }\end{array}$ & $39.6 \pm 0.5$ & $38 \pm 1.5$ & $34.8 \pm 4^{*}$ & $31.3 \pm 4.9^{*}$ \\
\hline Age, (years) & $28.7 \pm 4.9$ & $30.7 \pm 7.1$ & $34.3 \pm 8.2$ & $26.1 \pm 6.6$ \\
\hline $\begin{array}{c}\text { Body } \\
\text { mass index }\end{array}$ & $24.2 \pm 2.3$ & $25.8 \pm 1.6$ & $25.2 \pm 4.8$ & $24.9 \pm 6.7$ \\
\hline $\begin{array}{c}\text { Fetal } \\
\text { weight (g) }\end{array}$ & $3602 \pm 409$ & $3274 \pm 732$ & $2383.6 \pm 858.2^{*}$ & $\begin{array}{c}1367.6 \pm \\
863.1^{*}\end{array}$ \\
\hline $\begin{array}{l}\text { Placental } \\
\text { mass (g) }\end{array}$ & $476.8 \pm 62.5$ & $417.1 \pm 110.1$ & $338.1 \pm 121.9$ & $\begin{array}{c}249.6 \pm \\
133.6^{*}\end{array}$ \\
\hline $\begin{array}{c}\text { Placental/ } \\
\text { fetal weight } \\
\text { index }\end{array}$ & $0.13 \pm 0.02$ & $0.13 \pm 0.03$ & $0.15 \pm 0.04$ & $0.20 \pm 0.09$ \\
\hline
\end{tabular}

is typical for longstanding uteroplacental hypoxia-excessive syncytial knotting-was revealed. 3 cases of severe PE with IUGR displayed the signs of terminal villous hypoplasia. Placental multiple infarcts were observed in 6 cases of severe PE and in 8 cases of severe PE with IUGR.

\subsection{Immunohistochemistry}

GLUT-1 syncytial expression (Figure 1) in the terminal villi of severe PE cases placentas (both with and without IUGR) was significantly lower in compare to control group (Figure 2) ( $p=0.004, p=0.003$, accordingly) (Figure 3). GLUT-1 terminal villi endothelial expression in moderate PE and severe PE with IUGR groups was insignificantly lower in compare to control ( $\mathrm{p}$ $>0.05$ ). The only significant difference in GLUT-1 endothelial expression was observed in severe PE group without IUGR ( $p=0.01)$. Both syncytial and endothelial GLUT-3 expression (Figures 4 and 5) level was insignificantly lower in all the study groups in compare to control ( $p>0.05$ ). GLUT-1 syncytial-endothelial gradient was lower in all the study groups in compare to control, but the difference was only significantly in severe PE with IUGR group (1.27 versus $1.47, \mathrm{p}=0.03)$. GLUT3 syncytial-endothelial gradient was also lower in all the study groups but without significance $(p>0.05)$.

\section{DISCUSSION}

Glucose is essential for the development of the fetus. After oxygen, which is absolutely necessary for fetal development, glucose is the second important substrate for fetal growth and the only one that can be metabolized anaerobically. The growing glucose requirements of the fetus throughout pregnancy must be met by increases in placental transport capacity, which is mediated by the specific transmembrane glucose transporters (GLUTs).

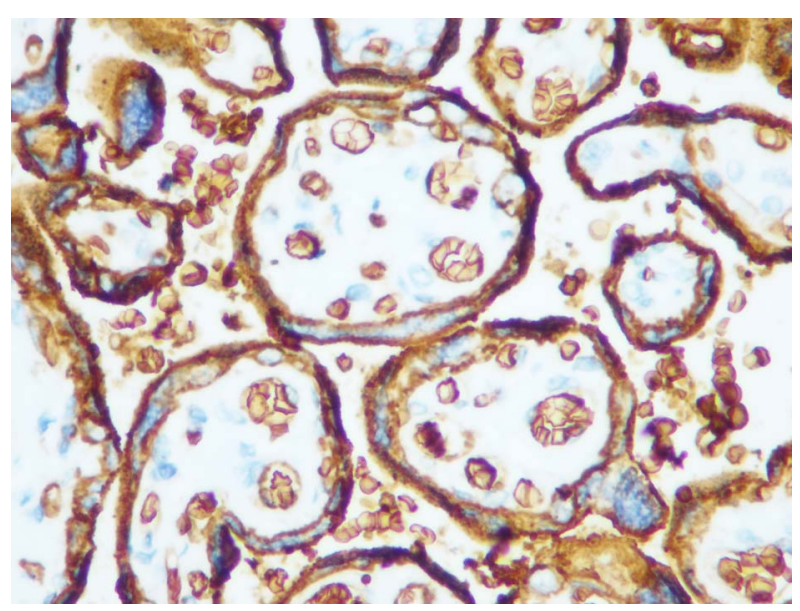

Figure 1. GLUT-1 expression in the placental terminal villisevere PE without IUGR ( $\times 40$ magnification). 


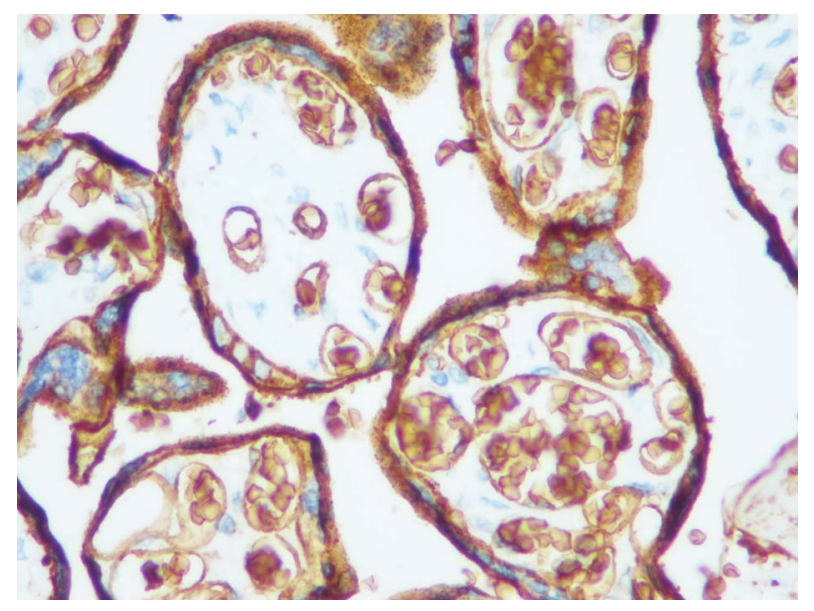

Figure 2. GLUT-1 expression in the placental terminal villicontrol ( $\times 40$ magnification).

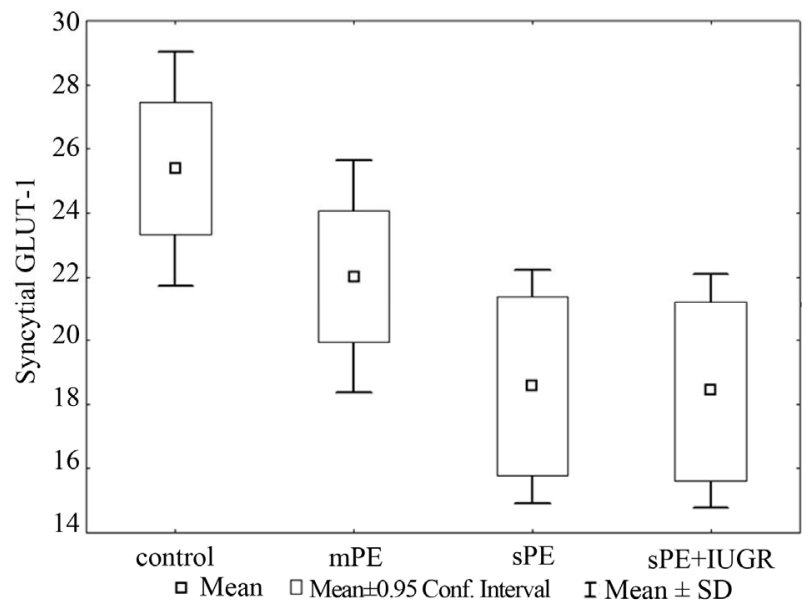

Figure 3. GLUT-1 syncytial expression levels.

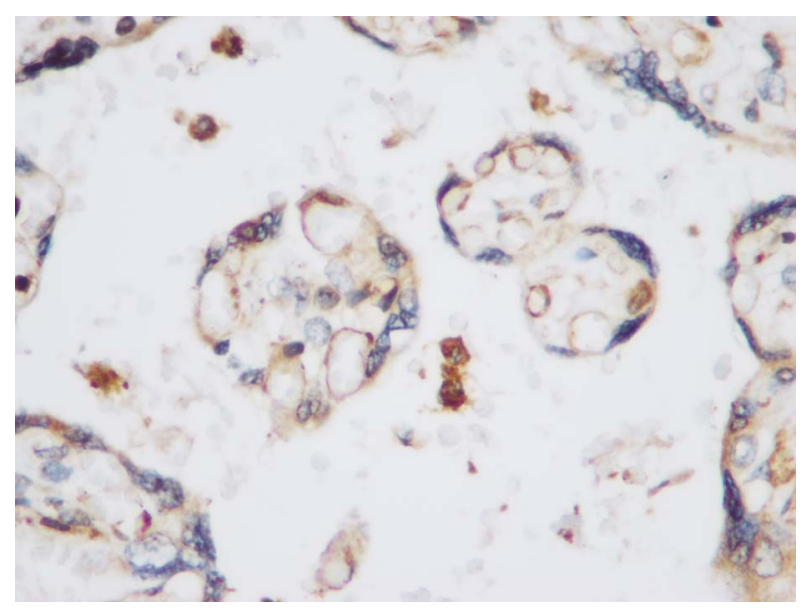

Figure 4. GLUT-3 expression in the placental terminal villisevere PE with IUGR $(\times 40$ magnification).

GLUTs are the members of sodium-independent transmembrane proteins that provide intracellular glucose transport [6]. A group of these molecules consists of at

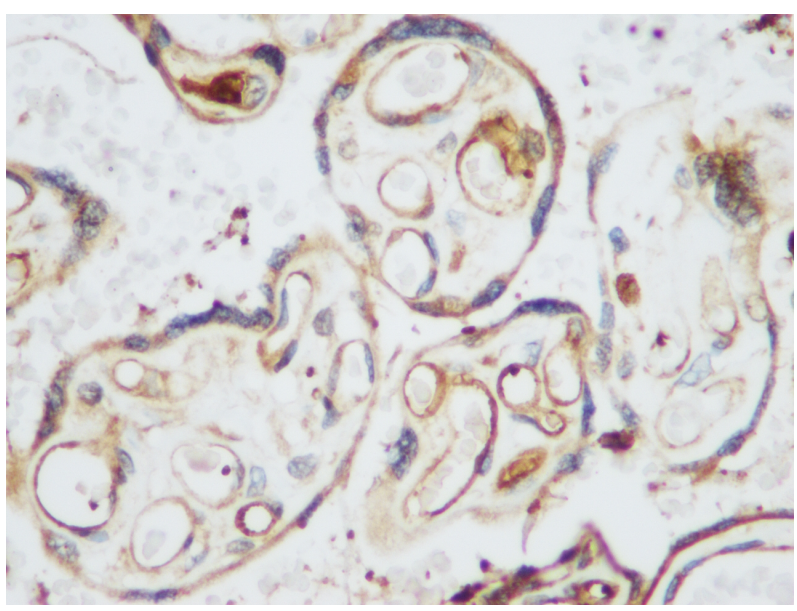

Figure 5. GLUT-3 expression in the placental terminal villicontrol ( $\times 40$ magnification).

least 12 isoforms. While many of these isoforms have been identified in human placental tissue, it is important to note that the only transporter present in the syncytium as a functional protein near term is the GLUT-1 isoform. In an experiment on rabbits [7] immunohistochemistry indicated the distribution of GLUT-1 was observed mainly to the surface of the outer trophoblasts, whereas GLUT-3 mainly localized to the basal site of the inner trophoblasts and fetal blood vessels, suggesting that glucose is transported through GLUT-1 from the maternal blood stream for use as a placental fuel and for further transport through GLUT-3 to the fetal circulation.

According to the data maximal GLUT-1 syncytial expression is seen near term $[8,9]$. It is also important to note that GLUT-1 concentration in the syncytial microvilli is 3 times higher than in syncytial basal membrane. This asymmetry reflects GLUT-1 transport activity, because basal membrane is thought to be a key limiting step in transplacental glucose transport [6]. We revealed GLUT-1 syncytial expression in control and all the study groups and it is important to note that GLUT-1 syncytial expression in the terminal villi of severe PE cases (both with and without IUGR) was significantly lower in compare to control group. Study suggests, that clear hypoxia increases glucose transporter expression in the trophoblast cells is to $[10,11]$. We revealed no difference between GLUT-1 terminal villi endothelial expression in moderate PE and severe PE with IUGR and control group. The only significant difference in GLUT-1 endothelial expression was observed in severe PE group without IUGR. Therefor the nature of GLUT-1 decreased syncytial and endothelial expression in severe PE cases is still unclear in additional studies should be performed to reveal it.

Conflicting information regarding expression of GLUT3 protein in the human placenta has been reported and the localization and pattern of expression of GLUT-3 
protein across gestation has not been clearly defined. Some data suggest that GLUT-3 expression in term and near term pregnancy placenta is absent in syncytiotrophoblast, other-that that it is localized mainly in the syncytial basal membrane $[7,12,13]$. Recent study reports that immunohistochemistry localizes GLUT-3 expression to the syncytiotrophoblast although there is also expression in the cytotrophoblast and to some degree in the endothelium. Western blotting shows that GLUT-3 is expressed in the microvillous but not basal membranes of the syncytium [14]. Other experimental study shows that in the placentas of rats under chronic maternal restraint stress placental GLUT-1 protein levels decreases whereas GLUT-3 protein levels slightly increases [15]. In the study we report about GLUT-3 both syncytial and endothelial expression in the near term and term placental terminal villi. Both syncytial and endothelial GLUT-3 expression level was insignificantly lower in all the study groups in compare to control.

\section{CONCLUSION}

Downregulated GLUT-1 expression in syncytiotrophoblast of the terminal villi could play a role in IUGR development in cases of severe preeclampsia. But the nature of GLUT-1 decreased syncytial and endothelial expression in severe PE cases is still unclear in additional studies should be performed to reveal it. Other study should be performed to establish whether GLUT-3 is expressed in syncytium of the preterm and term placental terminal villi or not. Also the role of GLUTs in preeclampsia development needs to be established.

\section{ACKNOWLEDGEMENTS}

The study is partly supported by the President of Russia Grant MK 1294.2012.7

\section{REFERENCES}

[1] Lo, J.O., Mission, J.F. and Caughey, A.B. (2013) Hypertensive disease of pregnancy and maternal mortality. Current Opinion in Obstetrics \& Gynecology, 25, 124-132. doi:10.1097/GCO.0b013e32835e0ef5

[2] Grebennik, T.K. and Pavlovich, S.V. (2011) Possibilities for prediction of preeclampsia. Gynecology and Obstetrics, 6, 17-21.

[3] Kafkasli, A., Turkcuoglu, I. and Turhan, U. (2013) Maternal, fetal and perinatal characteristics of preeclampsia cases with and without abnormalities in uterine artery Doppler indexes. Journal of Maternal-Fetal and Neonatal Medicine, 26, 936-940. doi:10.3109/14767058.2013.766688

[4] Zamudio, S., Torricos, T., Fik, E., Oyala, M., Echalar L., Pullockaran, J., Tutino, E., Martin, B., Belliappa, S., Balanza, E. and Illsley, N.P. (2010) Hypoglycemia and the origin of hypoxia-induced reduction in human fetal growth.
PLoS ONE, 5, e8551. doi:10.1371/journal.pone.0008551

[5] Vardhana, P. and Illsley, N. (2002) Transepithelial glucose transport and metabolism in BeWo choriocarcinoma cells. Placenta, 23, 653-660. doi:10.1053/plac.2002.0857

[6] Baumann, M.U., Deborde, S. and Illsley, N.P. (2002) Placental glucose transfer and fetal growth. Endocrine, 19, 13-22. doi:10.1385/ENDO:19:1:13

[7] Khan, H., Kusakabe, K.T., Wakitani, S., Hiyama, M. and Kiso, Y.J. (2011) Quantitative expression and immunohistochemical detection of glucose transporters, GLUT1 and GLUT3 in the rabbit placenta during successful pregnancy. The Journal of Veterinary Medical Science, 73, 11771183.

[8] Aleksandrova, N.V., Dubova, E.A., Baev, O.R., Shchegolev, A.I. and Sukhikh, G.T. (2012) Expression of carbohydrate metabolism markers in full-term spontaneous and induced pregnancy. Bulletin of Experimental Biology and Medicine, 153, 540-544. doi:10.1007/s10517-012-1762-0

[9] Jansson, T., Wennergren, M. and Illsley, N.P. (1993) Glucose transporter protein expression in human placenta throughout gestation and in intrauterine growth retardation. The Journal of Clinical Endocrinology \& Metabolism, 77, 1554-1562. doi:10.1210/jc.77.6.1554

[10] Baumann, M., Zamudio, S. and Illsley, N. (2007) Hypoxic upregulation of glucose transporters in BeWo choriocarcinoma cells is mediated by hypoxia-inducible factor-1 (HIF-1). American Journal of Physiology, 293, 477-485. doi:10.1152/ajpcell.00075.2007

[11] Esterman, A., Greco, M.A., Mitani, Y., Finlay, T.H. and Ismail-Beigi, F. (1997) The effect of hypoxia on human trophoblast in culture: Morphology, glucose transport and metabolism. Placenta, 18, 129-136. doi:10.1016/S0143-4004(97)90084-9

[12] Hauguel-De Mouzon, S., Challier, J., Kacemi, A., Cauzac, M., Malek, A. and Girard, J. (1997) The GLUT3 glucose transporter isoform is differentially expressed within human placental cell types. The Journal of Clinical Endocrinology \& Metabolism, 82, 2689-2694. doi:10.1210/jc.82.8.2689

[13] Barros, L.F., Yudilevich, D.L., Jarvis, S.M., Beaumont, N. and Baldwin, S.A. (1995) Quantitation and immunolocalization of glucose transporters in the human placenta. Placenta, 16, 623-633. doi:10.1016/0143-4004(95)90031-4

[14] Browna, K., Hellerb, D.S., Zamudioc, S. and Illsleya, N.P. (2011) Glucose transporter 3 (GLUT3) protein expression in human placenta across gestation. Placenta, 32, 10411049. doi:10.1016/j.placenta.2011.09.014

[15] Mairesse, J., Lesage, J., Breton, C., Bréant, B., Hahn, T., Darnaudéry, M., Dickson, S.L., Seckl, J., Blondeau, B., Vieau, D., Maccari, S. and Viltart, O. (2007) Maternal stress alters endocrine function of the feto-placental unit in rats. American Journal of Physiology-Endocrinology and Metabolism, 292, E1526-E1533. doi:10.1152/ajpendo.00574.2006 


\section{LIST OF ABBREVIATIONS}

GLUT—glucose transporters;

PE-preeclampsia;

IUGR-intrauterine growth retardation. 